\title{
Multi-input Serial Adders for FPGA-like Computational Fabric
}

\author{
Herman Schmit \\ Google Research \\ schmit@google.com
}

\author{
Matthew Denton \\ Google Research \\ myuzaki@google.com
}

\begin{abstract}
In this paper, we present a new functional unit to replace the LUT in an FPGA-like computational fabric designed specifically for use to accelerate instance-specific sparse integer matrix multiplication. We use a suite of matrices, the VPR place-and-route tool, and modern architecture representations of the interconnect to examine this architectural idea. The new cell, called the K-ADD, increases density by $2.5 \times$ to $4 \times$, and increases performance by $8 \%$ to $30 \%$ by simultaneously increasing the clock rate and reducing the number of cycles to compute the product. This benefit magnifies the twoorders-of-magnitude advantage of using instance-specific matrix multipliers demonstrated in prior work. We investigate the cluster size, $N$, across multiple technology nodes. In that investigation, we see a sustained benefit to a larger cluster size $(N=8)$. This observation holds for both netlists mapped to a 6-LUT and to a 6ADD, which implies this behavior has more to do with the peculiar structure of these matrix multiplication netlists, not the different functional unit.
\end{abstract}

\section{CCS CONCEPTS}

- Hardware $\rightarrow$ Programmable logic elements; Hardware accelerators; Technology-mapping.

\section{KEYWORDS}

Field-programmable gate arrays; Instance-specific acceleration; Bitserial arithmetic; Sparse matrix multiplication; Reservoir computing;

\section{ACM Reference Format:}

Herman Schmit and Matthew Denton. 2022. Multi-input Serial Adders for FPGA-like Computational Fabric. In Proceedings of the 2022 ACM/SIGDA International Symposium on Field-Programmable Gate Arrays (FPGA '22), February 27-March 1, 2022, Virtual Event, CA, USA. ACM, New York, NY, USA, 7 pages. https://doi.org/10.1145/3490422.3502352

\section{INTRODUCTION}

FPGAs perform best when they leverage the ability to customize the solution to the problem. Most accelerators built on FPGAs are domain-specific: they take advantage of particular data movement or calculation that applies to a class of computational problems. A more radical approach is to provide a configuration customized to a problem instance [19]. Examples of instance-specific solutions in FPGAs include fixed-key cryptographic engines, where the constant

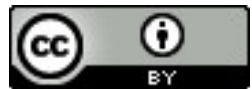

This work is licensed under a Creative Commons Attribution International 4.0 License.

FPGA '22, February 27-March 1, 2022, Virtual Event, CA, USA.

(c) 2022 Copyright is held by the owner/author(s).

ACM ISBN 978-1-4503-9149-8/22/02.

https://doi.org/10.1145/3490422.3502352 key is used to reduce the logic size and depth [13], and Boolean Satisfiability solvers that are specialized to a set of equations [23].

An FPGA-based solution was recently presented that uses an instance-specific configuration for a large sparse matrix-vector multiplication (SpMV) [7, 8]. That paper demonstrated one- and two-order of magnitude speedups in the inference speed of a recurrent system compared to GPU based solutions. In this particular application, the large matrix is permanently fixed. The overheads for design and configuration therefore can be amortized. That paper noted that the LUTs of the FPGA are underutilized: only three of the six inputs to each LUT were used, and one of those three inputs is local feedback for the state of serial adder.

This paper proposes a replacement functional unit for the LUT for fabrics designed specifically for bit-serial computation, and in particular for SpMV. We use a modern FPGA technology and interconnect to evaluate the benefit of the proposed customized functional unit (relative to a conventional LUT) and we consider the consequences to cluster size for the interconnect of the FPGA as a result.

The hypothesis that motivates this research is the following: Using bit- or digit-serial arithmetic components, connected via "programmable wires" and using instance-specific configurations is the most energy efficient way to perform sparse matrix computations on integers. This paper does not fundamentally prove this hypothesis, but it does build upon Denton et al.[7], which demonstrated the benefit of instance-specific configurations. In this paper, we present a specialized FPGA-like device that can significantly outperform a general-purpose FPGA.

\subsection{Contributions}

The contributions of this paper are as follows:

- We describe the K-ADD functional unit, which takes $K$ serial inputs and produces a single serial output representing the sum of the inputs.

- We show how using a 6-ADD serial adder can increase the area efficiency of the matrix multipliers by as much as $4 \times$ and can improve the clock rate and performance relative to a conventional 6-LUT based FPGA.

- We evaluate the cluster size $(N)$ using matrix multiplication netlists on conventional LUT-based FPGAs and our optimized fabric for four technology nodes: $16 \mathrm{~nm}, 7 \mathrm{~nm}, 5 \mathrm{~nm}$ and $4 \mathrm{~nm}$. We show different results than Nikolić et al.[16], which implies that the structure of the netlists differ from logic netlists in ways that may justify new interconnect investigations. 


\section{BACKGROUND}

\subsection{Sparse Matrix Multipliers and FPGAs}

The literature concerning the hardware architectures of sparse matrix arithmetic is vast. A good survey and bibliography can be found in Gao et al.[10]. The chief differentiator in most such hardware architectures for sparse computation are the techniques used to coordinate the elements from the sparse operands such that only a pair of non-zero operands are ever routed to a multiplieraccumulator. The point is to not waste energy and execution time on zeros. These architectures are also distinguished by the format they use to compress the matrix and matrix or vector to not waste RAM space.

A recent work [22] focusses on improving data locality for the inner and outer products when performing the SpGEMM operation. This data locality increases the data reuse and reduces the access to DRAM, which reduces power. It also condenses the sparse matrices internally to a more dense representation to allow for massive parallel multiplications.

In Denton et al.[7], the authors take a different approach to the the problem of connecting non-zero multiplicands of the matrix with the multiplicands in the vector. A programmable interconnect is used to wire operands directly from producer to consumer. The construction process is summarized in this way:

(1) Transform the elements in the matrix to a canonical signed digit representation [2] to reduce the number of ones in the matrix.

(2) Construct a tree of bit-serial adders to accumulate all of the vector inputs that exist in each bit position of each column of the transformed matrix. Each bit-serial adder has a registered output, which means that the depth of this tree grows logarithmically with the maximum number of bits in any column in the matrix. The number of 2-input adders required for the entire SpMV operation corresponds with the number of ones in the transformed matrix.

(3) Accumulate the bits in each column to produce a serialized result for each column.

(4) This design is placed and routed in an large FPGA, which determines the clock rate of this configuration.

It should be noted that the sparsity that is exploited in this implementation exists only in the matrix. The vector operand can be sparse or dense. Energy will be saved if the vector is sparse, but not area and not time. It is also worth noting that this method exploits both the element sparsity (the number of locations in the matrix that have a zero) and also the "bit-sparsity" (the number of ones in each number in the matrix). Bit-sparsity is increased by the canonical signed digit representation. It is also true that this approach requires the whole matrix to fit to a given FPGA.

As a result of the specialization to the one matrix, and as a result of the logarithmic depth of the tree, this technique provides a 2-3 order of magnitude reduction in multiplication latency relative to GPUs in the same technology node. This analysis was conducted against using state-of-the-art sparse libraries [9]. GPUs are not architected for very sparse matrices, so Denton et al.[7] compared against a proposed sparse accelerator [20] and still obtained a $4-47 \times$ reduction in multiplication latency.
This methodology produces a peculiar fanout distribution in the netlist: the primary input nets have a huge fanout, and every other output has a fanout of one because they are all in a tree. The critical path is almost always the input to the very large fanout. We add two pipeline stages on each primary input, which mitigates but does not eliminate this problem.

\subsection{LUTs and Clusters}

Historically, FPGA LUT size, measured by the number of inputs $(K)$, has increased from four to six (with some support for even bigger functions under limited circumstances.) Smaller $K$ is more area efficient, but larger $K$ consolidates more of a typical timing path into a high-speed logic element and less in the delay of the routable interconnect, which improves performance [1].

The UltraScale lookup table [21] can implement two functions of five inputs, as long as the two functions share all their inputs. This allows the sum and carry functions from a 2-input bit serial adder to map to a single LUT, as shown in Figure 1. The carry output can be registered, and fed back to the carry and sum functions within the same CLB. The sum function is also registered, and passed to subsequent terms in tree of adders. In Denton et al.[7], the number of bit-serial adders required by the matrix multiplication almost exactly equal to the number of LUTs, which is evidence that this is the way Vivado packs the logic.

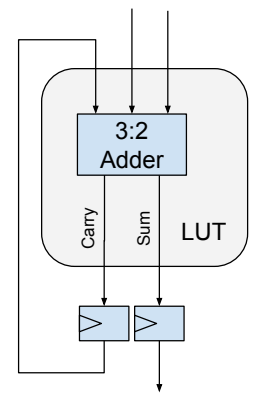

Figure 1: Ultrascale LUT Mapping of Bit Serial Adder

This mapping leaves three of the inputs of the UltraScale 6-LUT unused. The Agilex ALM [12] has even more input flexibility left unutilized. Large LUTs consume a lot of area, and providing for the routing of a large number of inputs consumes additional area.

Commercial FPGAs have created a hierarchical interconnect in the form of clusters of $N$ logic elements with especially high connectivity. These clusters, known commercially as the Configurable Logic Block (CLB) or the Logic Array Block (LAB), are designed to support the most interconnected portions of the netlist, and to enable efficient high-fanout nets. It is unclear whether the netlists generated by our matrix multiplication problem match the scaling behavior of most gate-level netlists, and therefore it is unclear whether the cluster as previously developed has value.

Given the changing characteristics of metal technology, Nikolic et al.[16] has shown that the large cluster sizes of earlier FPGAs may 
no longer be the optimal. That paper shows the optimum cluster size is between $N=4$ and $N=8$, until the $4 \mathrm{~nm}$ technology node, when a cluster size of $N=8$ becomes clearly inferior to $N=4$. In light of both netlist and technology changes, this paper will investigate the impact of the cluster size on area and performance.

\section{THE K-ADD FUNCTIONAL UNIT}

A two-input serial adder, as illustrated in Figure 1, can be generalized to accept $K$ inputs. Like the two-input case, this generalization will produce the least significant bit of the sum of the serialized inputs every cycle and maintain the more significant bits of the summation internally. We will refer to this generalization as a $\mathrm{K}-$ ADD.

Figure 2 shows a number of possible implementations of the $\mathrm{K}-\mathrm{ADD}$. It is always possible to build a $\mathrm{K}-\mathrm{ADD}$ using two other K-ADD units with $K 1$ and $K 2$ such that $K=K 1+K 2$ and use a final 2-ADD to add the results. $K=4$ and $K=6$ illustrate this structure in the figure. As a result, a K-ADD unit will require $K-1$ full adder cells and at most $K-1$ registers.

But alternatives may implement the same logic with possibly better delay results. A 5-ADD could be built from a 2-ADD and a 3-ADD, but that would lead to a critical path of three full adders and would require four registers. The solution in Figure 2 for $K=5$ shows an alternative that can reduce the critical path delay by one adder and save an internal register.

Like K-LUTs, the inputs to a K-ADD can be unused by tying off to zero. Since the function of each input is the same, input permutation can be performed to optimize delay, which VPR does not currently do. We expect that commercial tools rely on this property. This property is relied upon to exploit direct LUT-to-LUT connections [17].
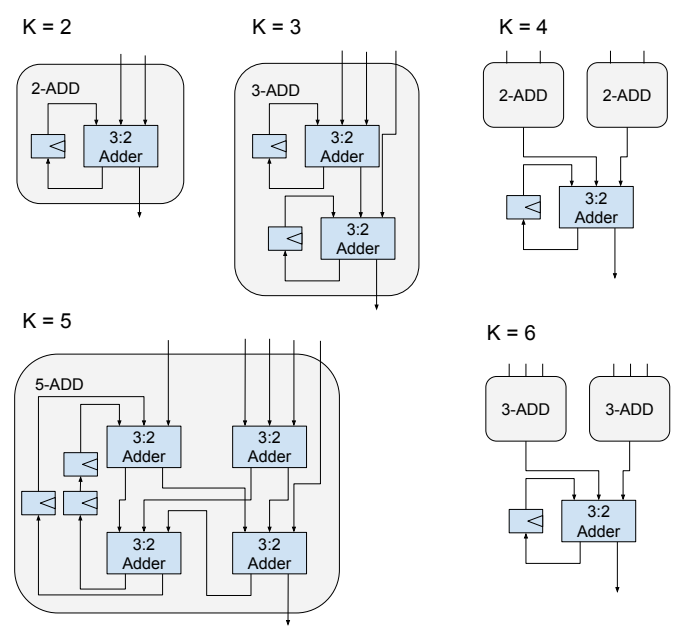

Figure 2: $\mathrm{K}-\mathrm{ADD}$ Logic for $\mathrm{K}=2,3,4,5,6$

Because it increases the amount of partial product reduction at each cycle, increased $K$ reduces the depth of the tree and therefore improves the overall design performance.

In this experiment, we are replacing all of the 6-LUTs of the FPGA with 6-ADD cells, which makes this device specialized to the problem of integer arithmetic. Such a device is not useful for general purpose logic replacement. We envision a system built out of 6ADD cells and programmable logic as a specialized IP core within a larger system, where that larger system is an ML accelerator, or even a larger general-purpose FPGA.

The 6-ADD should require approximately 400 transistors, whereas a 6-LUT requires approximately 700 transistors (assuming they are built on 6T SRAM cells and transmission gate based multiplexors). The smaller size of the 6-ADD would reduce the length of some number of routing wires, and thereby improve performance. However, accurately considering a differently sized functional unit would require a full re-design of the interconnect topology, which would confuse the analysis presented here.

\section{EVALUATION METHODOLOGY}

Our evaluation uses the VPR place-and-route tool[14] within the VTR flow[15]. The inputs to the VPR tools are BLIF files [18], which we generate directly from sparse matrix definitions. For the 6-LUT netlists, we map the matrix to a balanced tree of 2-input serial adders. As described in Section 2.2, commercial FPGAs can map the 2-input serial adder to a single LUT using multiple outputs, with the carry re-routed back to one of the LUT inputs. To capture this without relying on multi-output support in VPR, we represent the serial adders as 2-input XORs in BLIF. Since each stage of the tree is pipelined, the logic mapping tool has no freedom to consolidate the XOR functions into larger functions. For the 6-ADD, we take the same matrix and build a balanced tree of adders with as many as six inputs. The BLIF file for these netlists is represented as a 6-input XOR gate. Again, the fact that every stage is pipelined removes the tool's ability to repack the design.

Table 1 shows a suite of matrices with a variety of dimensions and sparsity. All of them are square, and all of them are eight-bit signed integers. These matrices were created by sweeping sparsity and dimension given the framework in Bianchi et al.[3] to find equivalent or better classification accuracy than the publication. This process was described in Denton et al.[8].

Table 1: Matrix Test Suite with Logic Reduction and Latency Results

\begin{tabular}{c|cr|cc|c} 
Name & Size & Sparsity & $\begin{array}{r}\text { 6-LUT } \\
\text { (Latency) }\end{array}$ & $\begin{array}{c}\text { 6-ADD } \\
\text { (Latency) }\end{array}$ & $\begin{array}{c}\text { Logic } \\
\text { Reduction }\end{array}$ \\
\hline $\mathrm{m} 1$ & 512 & $75 \%$ & $\begin{array}{c}261449 \\
(27)\end{array}$ & $\begin{array}{c}60677 \\
(23)\end{array}$ & $4.3 \times$ \\
\hline $\mathrm{m} 2$ & 256 & $85 \%$ & $\begin{array}{c}39577 \\
(26)\end{array}$ & $\begin{array}{c}10987 \\
(22)\end{array}$ & $3.6 \times$ \\
\hline $\mathrm{m} 3$ & 256 & $80 \%$ & $\begin{array}{c}52033 \\
(26)\end{array}$ & $\begin{array}{c}13113 \\
(23)\end{array}$ & $4.0 \times$ \\
\hline $\mathrm{m} 4$ & 2048 & $99 \%$ & $\begin{array}{c}166112 \\
(25)\end{array}$ & $\begin{array}{c}60889 \\
(22)\end{array}$ & $2.7 \times$ \\
\hline $\mathrm{m} 5$ & 512 & $80 \%$ & $\begin{array}{c}208879 \\
(27)\end{array}$ & $\begin{array}{c}51318 \\
(23)\end{array}$ & $4.1 \times$ \\
\hline $\mathrm{m} 6$ & 512 & $85 \%$ & $\begin{array}{c}156612 \\
(26)\end{array}$ & $\begin{array}{c}38379 \\
(23)\end{array}$ & $4.1 \times$ \\
\hline
\end{tabular}

Table 1 also shows the logic cell counts (for both 6-LUT vs 6-ADD) and the latency of the total matrix multiplication, as formulated in Denton et al.[7]. The logic reduction ranges from more 
Table 2: Architecture Files: Dimensions and Capacities

\begin{tabular}{rrr|rr}
$\mathrm{N}$ & $\mathrm{X}$ & $\mathrm{Y}$ & Logic Cells & IOs \\
\hline 2 & 235 & 561 & 260,494 & 3168 \\
4 & 234 & 281 & 258,912 & 4088 \\
8 & 235 & 141 & 259,096 & 5952 \\
16 & 203 & 81 & 254,064 & 8960
\end{tabular}

than a factor of $4.3 \times$ on netlist $\mathrm{m} 1$, to a factor of $2.7 \times$ on netlist $\mathrm{m} 4$. There seems to be some correlation between sparsity and benefit from the 6-ADD cell. In a very sparse matrix there are fewer matrix columns with enough terms to fully populate multiple levels of 6-ADD cells. In the $\mathrm{m} 4$ matrix, for example, each column has about 20 non-zero entries (1\% of 2048), and only half of the bits in those non-zero entries will be ones. Therefore, there are on average 10 non-zero bits per column-bit, which would be an inefficient use of the 6-ADD. This could be improved by using a construction process to allow accumulations of different bits in a column (with appropriate register delays) prior to the final accumulation. This idea is not investigated in this paper.

Asymptotically, replacing the entire fabric of UltraScale LUTs with $6-A D D$ s should reduce the logic cell count by $5 \times$, because a 6-ADD comprises five 2-input serial adders. Also asymptotically, we would expect the depth of the trees to be reduced by $\log 6 / \log 2$ or approximately $2.5 \times$.

The latency in Table 1 is computed by using Equation 5 in [7], generalized for the K-ADD and shown below as Equation 1. Equation 1 includes two pipeline stages for the primary input, and a register for the primary input and output. The values in Table 1 are based on the activation (as well as the weights) being eight-bit quantities.

$$
\text { Latency }=B W_{i}+B W_{w}+\left\lceil\log _{K} R\right\rceil+2+2
$$

Where $B W_{i}$ and $B W_{w}$ are the bitwidth of the input and weights respectively, and $R$ is the maximum number of non-zero entries any column in the matrix. Total latency is dominated by $B W_{i}+B W_{w}$ which is 16 cycles. The total system performance will be the product of this latency and the clock period attained by these netlists.

To obtain those clock rates, we used a recent version of VPR ${ }^{1}$ and large instances of the best architectures generated by [16]. Table 2 shows the architecture files generated for these experiments, using cluster sizes $(\mathrm{N})$ of $2,4,8$, and 16 . The the methodology created in [16] does not generate a square cluster. For small $\mathrm{N}$, the cluster is wider than it is tall, and for large $\mathrm{N}$, the cluster is taller than it is wide. The dimensions of the array have be modified to keep the physical array as square as possibly, while still providing approximately the same logic capacity. Architectures of these dimensions were generated for four technology nodes: $16 \mathrm{~nm}, 7 \mathrm{~nm}, 5 \mathrm{~nm}$ and $4 \mathrm{~nm}$. We use the best interconnect topologies for each technology node, as reported in [16]. We also use exactly the same technology models as [16], which is based on versions of the ASAP7 [5] and PMT [4] predictive models.

The greater logic depth within the 6-ADD versus the 6-LUT means it has higher delay. To estimate this, we have designed a

\footnotetext{
${ }^{1}$ Accessible at https://github.com/verilog-to-routing/vtr-verilog-to-routing/commit/ 110bae4ae127ed1a67fcf6c1b38f8a6ff9e24e46
}

Table 3: 6-LUT and 6-ADD Delays

\begin{tabular}{l|rr} 
Process & 6-LUT Delay [16] & 6-ADD Delay \\
\hline $16 \mathrm{~nm}$ & $94 \mathrm{ps}$ & $315 \mathrm{ps}$ \\
$7 \mathrm{~nm}$ & $68 \mathrm{ps}$ & $228 \mathrm{ps}$ \\
$5 \mathrm{~nm}$ & $64 \mathrm{ps}$ & $214 \mathrm{ps}$ \\
$4 \mathrm{~nm}$ & $64 \mathrm{ps}$ & $214 \mathrm{ps}$
\end{tabular}

6-ADD cell using a $7 \mathrm{~nm}$ standard cell library and then used the same performance scaling rules as applied to the LUT in [16]. The comparative delays for 6-LUT and 6-ADD are shown in Table 3. These delays are incorporated into the 6-ADD architecture files for VPR.

\section{RESULTS}

\subsection{6nm Results}

In this first experiment, we evaluate the mappings of all of the matrices on all instances of the 6-ADD and 6-LUT architectures in the $16 \mathrm{~nm}$ technology node. This technology node enables us to compare against the Ultrascale Plus analysis in [7].

The resulting best-case frequencies (considering different cluster sizes) for the four matrices that can all be mapped to all of the architectures is shown in Figure $3 .^{2}$ In most cases, the frequency is moderately higher for the 6-ADD designs, even with the additional delay for the functional unit.

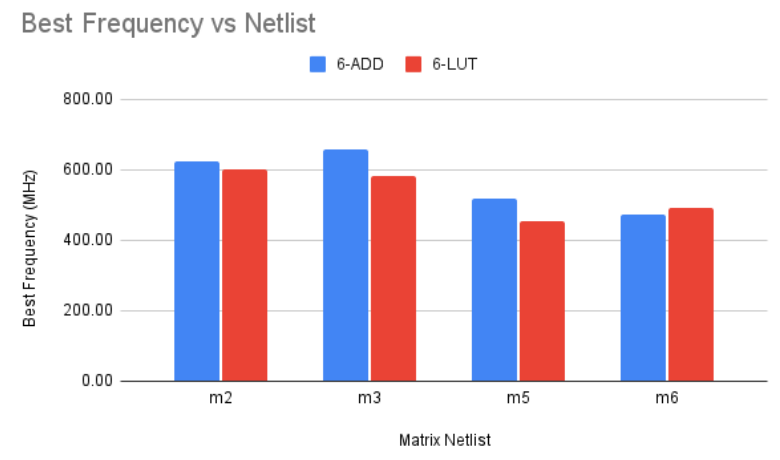

Figure 3: 16nm Comparison: Best Case Frequency vs Matrix Netlist

Another way to look at this data is shown in Figure 4, which plots frequency versus logic cell count for all the implementations (including some for matrices $\mathrm{m} 1$ and $\mathrm{m} 4$.) In this plot, it is clear that the frequency benefit of the $6-\mathrm{ADD}$ is primarily attributed to the smaller netlist size of the equivalent matrices. In fact, it seems that the 6-LUT designs seem to exhibit less frequency reduction as the designs grow. This graph also resembles the Ultrascale plots of frequency vs size in [7]. The peak frequency in [7] is nearer to $800 \mathrm{MHz}$, and falls away much more slowly. VPR is not as performance and memory capable as Vivado. Additionally, the architecture files in

\footnotetext{
${ }^{2}$ Matrix $\mathrm{m} 1$, when compiled to the 6-LUT, does not fit into any of the archtitectures because of logic capacity. Matrix $\mathrm{m} 4$ does not fit into some of the architectures because of IO requirements. They are therefore excluded from this graph.
} 
[16] limit the number of inter-cluster wires to a single layer for vertical and horizontal dimensions, which is unlikely true for UltraScale Plus.

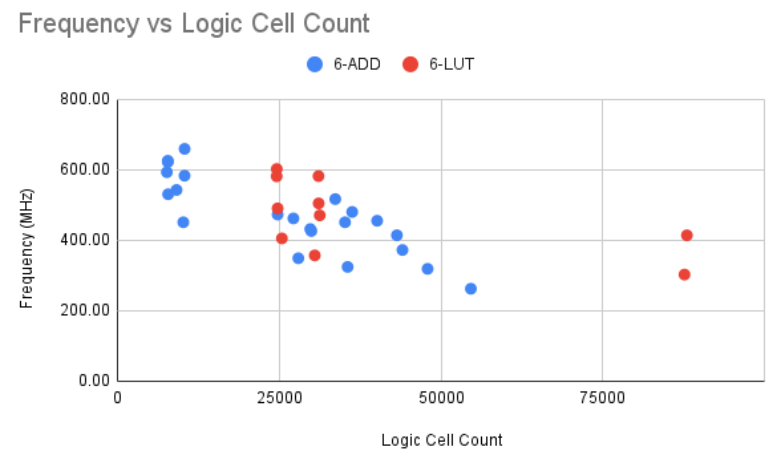

Figure 4: Frequency vs Logic Cell Count

The critical path of many of these designs is the initial broadcast of the input operands to the first stage of partial product reductions. We have added two additional pipeline stages at the entry layer to mitigate some of this problem, but the right fix to this problem requires placement-dependent register replication. VPR does not support these internal optimizations, which could also explain the difference in design scaling versus Vivado.

To understand this frequency scaling, Figure 5 plots peak routing channel utilization versus logic cell count. Peak routing channel utilization is the occupancy of the wires in the most highly utilized routing channel and is a noisy indicator of congestion. The 6-ADD designs, because they are more pin intensive, push the utilization up much more rapidly. This is what you might expect in a logic design mapped to 6-LUTs. The 6-LUT designs have very low pin counts by construction, and the fact that the channel occupancy never gets over $40 \%$ is an indication that these netlists are not using the interconnect provisioned for them. This may enable them to maintain a reasonable frequency for larger design sizes.

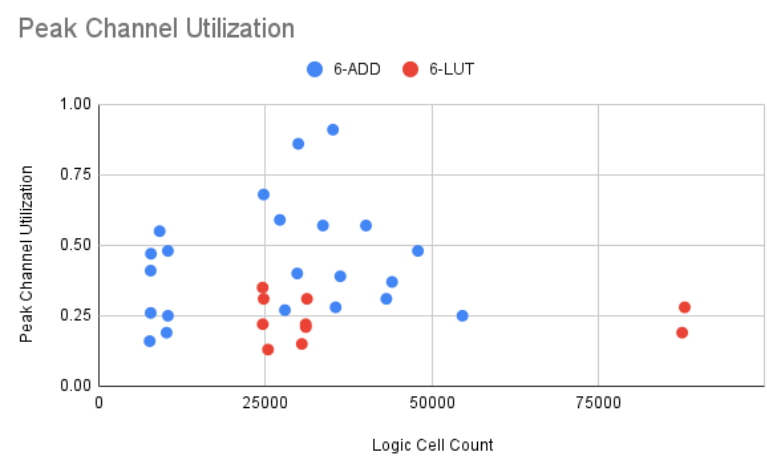

Figure 5: Peak Channel Utilization vs Logic Cell Count

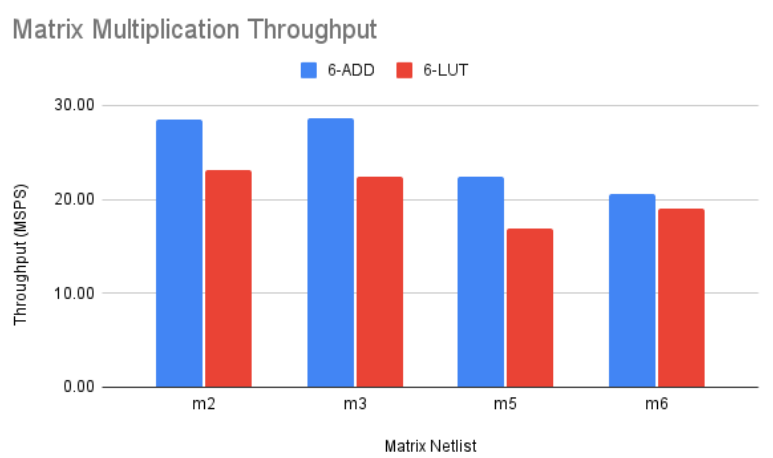

Figure 6: $16 \mathrm{~nm}$ Comparison: Matrix Multiplication Throughput (Inverse of the Product of Latency Cycles and Clock Period)

\subsection{Optimal Cluster Size}

Figure 6 shows the total performance of the four netlists that fit on all architectures. This is the best frequency from Figure 3 divided by the netlist latency. The performance benefit of the 6-ADD ranges from $8 \%$ on $\mathrm{m} 6$ to $33 \%$ on $\mathrm{m} 3$.

Figure 7 shows the average frequency across netlists $m 2, \mathrm{~m} 3, \mathrm{~m} 5$, and $\mathrm{m} 6$ for different cluster sizes in $16 \mathrm{~nm}$ technology. The 6-ADD design is fairly immune to variations in the cluster size, but the 6-LUT design definitely has a big degradation for the $N=8$ and $N=16$. In [16], the $N=4$ and $N=8$ cluster sizes were about the same in $16 \mathrm{~nm}$. Because $6-\mathrm{ADD}$ netlists more closely resemble the fanin and fanout characteristics of conventional netlists, the clustering is more beneficial.

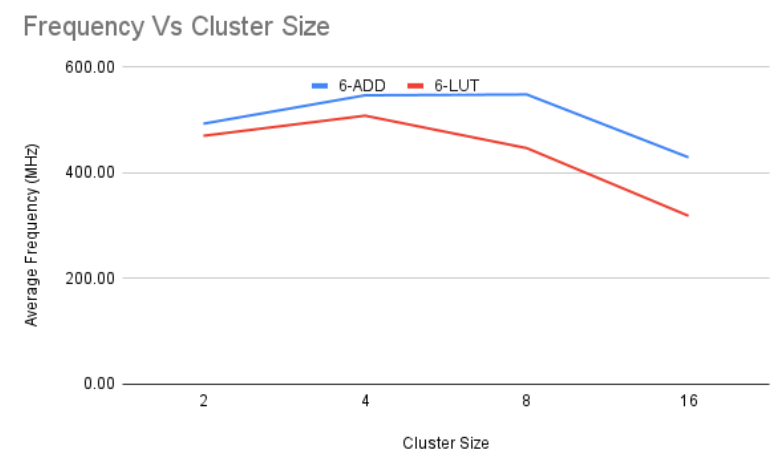

Figure 7: Frequency vs Cluster Size (16nm)

Figure 8 shows the average frequency for runs (with six different placement seeds) of the $\mathrm{m} 2$ and $\mathrm{m} 3$ matrix netlists for $16 \mathrm{~nm}, 7 \mathrm{~nm}$, $5 \mathrm{~nm}$ and $4 \mathrm{~nm}$. These figures are to contrast to Figure 13 in [16]. Like in that paper, there is no longer a clear frequency scaling between process nodes. In particular, frequency degrades from $5 \mathrm{~nm}$ to $4 \mathrm{~nm}$ due to the increasing interconnect resistance. The prior paper showed that $N=16$ was terrible for all process nodes, and an increasing trend to smaller cluster sizes being optimal in smaller 


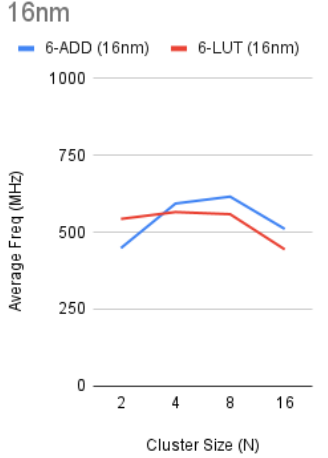

(a)

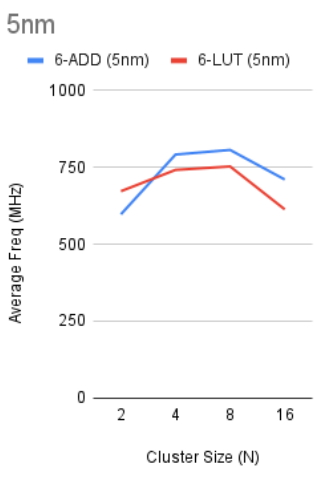

(c) (b)

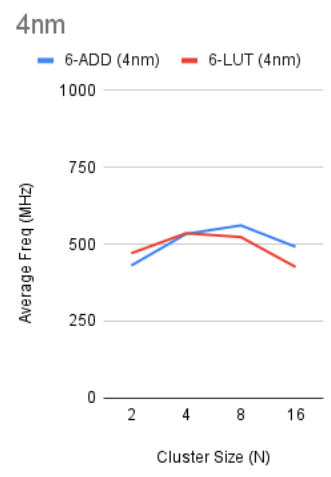

(d)

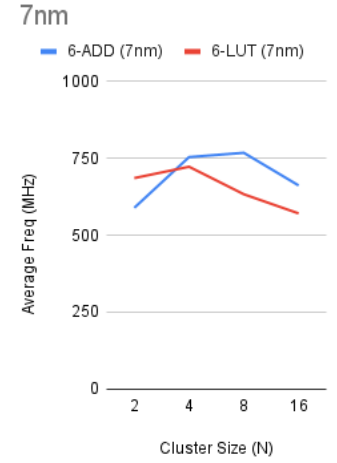

Figure 8: Frequency vs Cluster Size (for $16 \mathrm{~nm}$, $7 \mathrm{~nm}$ 5nm, and 4nm)

process technologies. This is not so clear for either the 6-ADD or 6-LUT netlists. $N=8$ seems to be the optimal or near optimal cluster size. Because the 6-ADD and 6-LUT designs exhibit similar behavior, we hypothesize that the logic unit is not as important as the peculiar topological aspects of these matrix multiplication netlists. Specifically, because the critical path of these netlists is most often the primary input tree, the clustering could be useful in supporting the high fanout of those nets.

\section{DISCUSSION AND FUTURE WORK}

As stated in the introduction, we are motivated by the hypothesis that serial arithmetic, constant propagation, and programmable wires is an extremely energy and silicon efficient way to do sparse integer matrix multiplication. Constant propagation fundamentally reduces the amount of work to perform a multiplication. We can leverage the sparsity of the matrix, defined as the percentage of nonzero elements in the matrix. We also leverage the sparsity within the number representations. Doing arithmetic serially minimizes the false switching present in most arithmetic unit implementations. Programmable interconnect allows us to build a matrix-specific configuration that can have logarithmic depth with the number of non-zeros in each matrix column, which is much better than the linear depth of systolic arrays for dense matrix multiplication.

This paper considered the adaptation of the functional unit in the context of an FPGA-optimized interconnect. But there are ways to extract even more efficiency. First, the connectivity of these netlists is bimodal: nets in the first stage of the tree have huge fanout, and each of the subsequent nets has a fanout of exactly one. This is very different than general logic netlists, and the interconnect would likely benefit from special resources for both types of nets. Second, we may consider replacing the single-bit wires with multi-bit wires to reduce the computation latency even further. This would more greatly amortize the configuration overhead of the interconnect.

In the software realm, an ability to retime the logic to eliminate critical paths on interconnect delays would make a significant improvement when searching for the optimum of the product of cycle time and cycles of latency. In addition, there appears to be significant redundancy in these networks. Finding a way to factor the common subexpressions could lead to density improvements, and possibly further clock rate improvements due to reduction of design size.

A limitation to the applicability of any instance-specific configuration is the slow configuration time of conventional FPGAs. This is not a problem in [7] because in reservoir computing the matrix is permanently fixed. But a device with high-speed reconfiguration could be applied in ML computations requiring multiple layers, and could allow for the time-multiplexing of a smaller physical fabric to emulate a larger one. There are many solutions to high speed reconfiguration $[6,11]$ with different tradeoffs. Obviously, moving configuration data requires energy. Multiple frames of configuration would increase the tile size and therefore the energy required to perform a computation. We hope to analyze these questions in future papers.

\section{CONCLUSION}

A new logic unit has been introduced, the K-ADD. This variant where $K=6(6-\mathrm{ADD})$ reduces the logic size of instance-specific configurations of matrix multipliers by a factor from $2.5 \times$ to $4 \times$. Because a 6-ADD does reduces more terms every cycle, the number of cycles of latency are also reduced. As a side-effect of the reduction in logic size, we have also measured a reduction in clock cycle time. The net improvement in performance is $8 \%$ to $33 \%$, which magnifies the two-orders of magnitude gains demonstrated in prior literature.

\section{ACKNOWLEDGMENTS}

Special thanks to Stefan Nikolić at EPFL for his help with VPR architecture files. Thanks to the reviewers who provided copious suggestions to improve this paper.

\section{REFERENCES}

[1] E. Ahmed and J. Rose. 2004. The effect of LUT and cluster size on deep-submicron FPGA performance and density. IEEE Transactions on Very Large Scale Integration (VLSI) Systems 12, 3 (2004), 288-298. https://doi.org/10.1109/TVLSI.2004.824300

[2] A. Avizienis. 1961. Signed-Digit Number Representations for Fast Parallel Arithmetic. IRE Transactions on Electronic Computers EC-10, 3 (1961), 389-400. https://doi.org/10.1109/TEC.1961.5219227

[3] F. M. Bianchi, S. Scardapane, S. Løkse, and R. Jenssen. 2020. Reservoir Computing Approaches for Representation and Classification of Multivariate Time Series. IEEE Transactions on Neural Networks and Learning Systems (2020), 1-11. 
[4] Yu Cao. [n.d.]. Predictive Technology Model. http://ptm.asu.edu/ (accessed Aug 26, 2020).

[5] Lawrence T. Clark, Vinay Vashishtha, Lucian Shifren, Aditya Gujja, Saurabh Sinha, Brian Cline, Chandarasekaran Ramamurthy, and Greg Yeric. 2016. ASAP7: A 7-nm finFET predictive process design kit. Microelectronics fournal 53 (2016), 105-115. https://doi.org/10.1016/j.mejo.2016.04.006

[6] A. DeHon. 1994. DPGA-coupled microprocessors: commodity ICs for the early 21st Century. In Proceedings of IEEE Workshop on FPGA's for Custom Computing Machines. 31-39. https://doi.org/10.1109/FPGA.1994.315596

[7] Matthew Denton and Herman Schmit. 2021. Direct Spatial Implementation of Sparse Matrix Multipliers for Reservoir Computing. CoRR abs/2101.08884 (2021) arXiv:2101.08884 https://arxiv.org/abs/2101.08884

[8] Matthew Denton and Herman Schmit. 2022. Direct Spatial Implementation of Sparse Matrix Multipliers for Reservoir Computing. In 2022 IEEE International Symposium on High Performance Computer Architecture (HPCA).

[9] Trevor Gale, Matei Zaharia, Cliff Young, and Erich Elsen. 2020. Sparse GPU Kernels for Deep Learning. arXiv:2006.10901 [cs.LG] https://arxiv.org/abs/2006. 10901

[10] Jianhua Gao, Weixing Ji, Zhaonian Tan, and Yueyan Zhao. 2020. A Systematic Survey of General Sparse Matrix-Matrix Multiplication. CoRR abs/2002.11273 (2020). arXiv:2002.11273 https://arxiv.org/abs/2002.11273

[11] Seth Copen Goldstein, Herman Schmit, Matthew Moe, Mihai Budiu, Srihari Cadambi, R. Reed Taylor, and Ronald Laufer. 1999. PipeRench: A Co/Processor for Streaming Multimedia Acceleration. In Proceedings of the 26th Annual International Symposium on Computer Architecture (Atlanta, Georgia, USA) (ISCA '99). IEEE Computer Society, USA, 28-39. https://doi.org/10.1145/300979.300982

[12] Intel. 2019 (accessed Aug 30, 2021). Intel Agilex Logic Array Block and Adaptive Logic Modules User Guide, UG-20204 (2019.11.14). https://www.intel.com/content/ dam/www/programmable/us/en/pdfs/literature/hb/agilex/ug-ag-lab.pdf.

[13] Jason Leonard and William H. Mangione-Smith. 1997. A case study of partially evaluated hardware circuits: Key-specific DES. In Field-Programmable Logic and Applications, Wayne Luk, Peter Y. K. Cheung, and Manfred Glesner (Eds.) Springer Berlin Heidelberg, Berlin, Heidelberg, 151-160.

[14] Jason Luu, Ian Kuon, Peter Jamieson, Ted Campbell, Andy Ye, Wei Mark Fang, and Jonathan Rose. 2009. VPR 5.0: FPGA Cad and Architecture Exploration Tools with Single-Driver Routing, Heterogeneity and Process Scaling. In Proceedings of the ACM/SIGDA International Symposium on Field Programmable Gate Arrays (Monterey, California, USA) (FPGA '09). Association for Computing Machinery,
New York, NY, USA, 133-142. https://doi.org/10.1145/1508128.1508150

[15] Kevin E. Murray, Oleg Petelin, Sheng Zhong, Jai Min Wang, Mohamed ElDafrawy, Jean-Philippe Legault, Eugene Sha, Aaron G. Graham, Jean Wu, Matthew J. P. Walker, Hanqing Zeng, Panagiotis Patros, Jason Luu, Kenneth B. Kent, and Vaughn Betz. 2020. VTR 8: High Performance CAD and Customizable FPGA Architecture Modelling. ACM Trans. Reconfigurable Technol. Syst. (2020).

[16] Stefan Nikolić, Francky Catthoor, Zsolt Tökei, and Paolo Ienne. 2021. Global Is the New Local: FPGA Architecture at 5nm and Beyond. In The 2021 ACM/SIGDA International Symposium on Field-Programmable Gate Arrays (Virtual Event, USA) (FPGA '21). Association for Computing Machinery, New York, NY, USA, 34-44. https://doi.org/10.1145/3431920.3439300

[17] Stefan Nikolić, Grace Zgheib, and Paolo Ienne. 2020. Straight to the Point: Intra- and Intercluster LUT Connections to Mitigate the Delay of Programmable Routing. In Proceedings of the 2020 ACM/SIGDA International Symposium on Field-Programmable Gate Arrays (Seaside, CA, USA) (FPGA '20). Association for Computing Machinery, New York, NY, USA, 150-160. https://doi.org/10.1145/ 3373087.3375315

[18] University of California Berkeley. 1992. Berkeley Logic Interchange Format (BLIF). https://docs.verilogtorouting.org/en/latest/downloads/ a79b6634b582f56c053f2abad923112a/blif.pdf. Accessed: 2021-09-07.

[19] Oliver Pell and Wayne Luk. 2008. Instance-specific Design. In Reconfigurable Computing: The Theory and Practice of FPGA-Based Computation, Scott Hauck and Andre Dehon (Eds.). Morgan-Kaufman, Chapter 22, 455-473.

[20] E. Qin, A. Samajdar, H. Kwon, V. Nadella, S. Srinivasan, D. Das, B. Kaul, and T. Krishna. 2020. SIGMA: A Sparse and Irregular GEMM Accelerator with Flexible Interconnects for DNN Training. In 2020 IEEE International Symposium on High Performance Computer Architecture (HPCA). 58-70.

[21] Xilinx. 2017 (accessed Aug 30, 2021). UltraScale Architecture CLB User Guide, UG574 (v1.5). https://www.xilinx.com/support/documentation/user_guides/ ug574-ultrascale-clb.pdf

[22] Zhekai Zhang, Hanrui Wang, Song Han, and William J. Dally. 2020. SpArch: Efficient Architecture for Sparse Matrix Multiplication. In 2020 IEEE International Symposium on High Performance Computer Architecture (HPCA). 261-274. https: //doi.org/10.1109/HPCA47549.2020.00030

[23] Peixin Zhong, Margaret Martonosi, and Sharad Malik. 2008. Boolean satisfiability: Creating solvers optimized for specific problem instances. In Reconfigurable Computing: The Theory and Practice of FPGA-Based Computation, Scott Hauck and Andre Dehon (Eds.). Morgan-Kaufman, Chapter 29, 613-636. 